\title{
Radiolabeled Lipiodol Therapy for Hepatocellular Carcinoma in Patients Awaiting Liver Transplantation: Pathology of the Explant Livers and Clinical Outcome
}

\author{
Bieke Lambert, ${ }^{1}$ Marleen Praet, ${ }^{2}$ Peter Vanlangenhove, ${ }^{3}$ Roberto Troisi, ${ }^{4}$ \\ Bernard de Hemptinne, ${ }^{4}$ Filip Gemmel, ${ }^{1}$ Hans Van Vlierberghe, ${ }^{5}$ and Christophe Van de Wiele ${ }^{1}$ \\ ${ }^{1}$ Division of Nuclear Medicine, ${ }^{2}$ Department of Pathology, ${ }^{3}$ Division of Interventional Radiology, \\ ${ }^{4}$ Department of Surgery, and ${ }^{5}$ Division of Gastro-Enterology, Ghent University Hospital, Ghent, \\ Belgium
}

\begin{abstract}
Background: Liver transplantation has become an important curative treatment option for hepatocellular carcinoma (HCC). Criteria for transplantation are strict and, therefore, it is crucial that patients awaiting transplantation do not suffer disease progression. One of the therapeutic options to achieve disease stabilization is neoadjuvant radiolabeled lipiodol treatment. This study aimed to document the dropout rate on the waiting list, the pathological findings on the explant livers, and the long-term outcome of patients treated with radionuclide therapy while awaiting transplantation. Methods: Patients eligible for transplantation were treated with $2.1 \mathrm{GBq}{ }^{131}$ I-lipiodol or $4.1 \mathrm{GBq}{ }^{188}$ Re-HDD/lipiodol by transfemoral catheterization of the hepatic arteries. Tumor necrosis was assessed in the explant livers and follow-up data, such as dropout from the waiting list, recurrence, and survival following transplantation were retrospectively documented. Results: In 5 of 22 explants, necrosis exceeded 90\%. Two patients died while on the waiting list (10\%) and 4 of 20 transplanted patients (20\%) suffered recurrent disease. The overall recurrence-free survival was 19.7 months (range, 1.75-56), with a mean follow-up of 20.1 months. Conclusion: Our data support the evaluation on larger patient numbers to confirm the benefit of radiolabeled lipiodol in candidates for liver transplantation who are suffering from HCC.
\end{abstract}

Key words: hepatocellular carcinoma, liver transplantation, iodine-131-lipiodol, rhenium-188-HDD/lipiodol, radionuclide therapy

\section{INTRODUCTION}

Hepatocellular carcinoma (HCC) is the most prevalent malignant primary liver tumor. It constitutes the 3rd-leading cause of cancer-related

Address reprint requests to: Bieke Lambert; Division of Nuclear Medicine, Ghent University Hospital; De Pintelaan 185, B-9000 Ghent, Belgium; Tel.: +32 9 2403028; Fax: +32 92403807

E-mail: Bieke.Lambert@Ugent.be deaths, is responsible for more than 500,000 deaths worldwide annually. ${ }^{1}$ Currently, the mainstay of potentially curative strategies for HCC consists of hepatectomy - either a partial liver resection or a total removal of the liver-followed by cadaver organ transplantation or by living related liver donation. Three major criteria should be met, in case partial liver resection is considered: the absence of metastatic spread, no bilobar disease, and an adequate liver function afterwards. ${ }^{2}$ The two latter criteria are not essential in 
case transplantation is an option. ${ }^{3}$ As HCC typically presents late in its natural course, the vast majority of patients are not suitable for liver resection.

Theoretically, liver transplantation is the optimum therapy for HCC, as it provides for the removal of the primary tumor, as well as the cirrhotic parenchyma, which is a seed-bed for the development of HCC. For advanced disease stages of $\mathrm{HCC}$, retrospective analyses have shown poor outcomes after transplantation, yielding unacceptable recurrence rates. ${ }^{3}$ Therefore, strict criteria concerning the dimensions and numbers of tumors were advocated, and, in wellselected patients, survival rates equalled those obtained in patients transplanted because of benign liver pathology. ${ }^{4-6}$ Because tumor size is a very important variable in predicting recurrence, it is essential that patients are transplanted before progression occurs. The waiting time for a classic liver transplantation in the Western world may cause a delay, which can allow the tumor to grow to stages that contraindicate transplantation ${ }^{7}$ and, therefore, several neoadjuvant strategies have been proposed. ${ }^{8}$

Chemoembolization is the most common neoadjuvant treatment, but no randomized, controlled studies have been reported so far to show an effect on the outcome. ${ }^{8,9}$ Using a nonselective arterial instillation, ${ }^{131}$ I-lipiodol has been found to be equally effective in tumor control but is far better tolerated than the classical chemoembolization by cisplatinum, lipiodol, and gelatin-sponge fragments in a group of inoperable, palliative patients. ${ }^{10}$ The initial studies using ${ }^{131}$ I-lipiodol in a neoadjuvant setting were conducted by Brans et al. and by Raoul et al. ${ }^{11,12}$

This study aims to report our experiences in using radiolabeled lipiodol for disease stabilization in patients awaiting liver transplantation. The dropout rate of patients on the waiting list, despite neoadjuvant treatment, was calculated. A pathological examination of the explant livers was performed in order to document antitumoral effects of therapy. Clinical follow-up data of the transplant patients were retrospectively collected and recurrence rates were estimated.

\section{MATERIALS AND METHODS}

\section{Patient Selection}

The diagnosis of HCC was established on the histological examination of biopsy specimens or on the radiological appearance of the lesions on imaging, eventually in combination with a rise in serum alpha-fetoprotein level. Patients were judged to be unresectable because of coexisting decompensated liver cirrhosis and/or multinodular HCC. Before listing for transplantation, metastatic spread was ruled out by bone scan, by computed tomography (CT) scan of the chest and brain. Patients undergoing radiolabeled lipiodol treatment gave written, informed consent. $\mathrm{Pa}-$ tients suffering Child $\mathrm{C}$ cirrhosis were not eligible for radionuclide treatment. Patients who had anticancer treatment other than radiolabeled lipiodol in their history were excluded from the analysis.

\section{Neoadjuvant Treatment}

The ${ }^{131}$ I-labeled lipiodol (Lipiocis ${ }^{\mathrm{TM}}$ ) was purchased at CIS Bio International (Gif-sur-Yvette, France) and, since 2002, ${ }^{188} \mathrm{Re}-\mathrm{HDD} / \mathrm{lipiodol}$ was used. Lyophilized kits containing a HDDchelator (4-hexadecyl-1-2, 9, 9-tetramethyl-4, 7diaza-1,10-decanethiol) were provided by the Seoul National University Hospital (Seoul, Korea) and ${ }^{188}$ ReHDD/lipiodol was synthesized, as described earlier by Lee et al. ${ }^{13}$ After selective hepatic catheterization, a mean activity of 2.1 $\mathrm{GBq}{ }^{131} \mathrm{I}$ or $4.1 \mathrm{GBq}{ }^{188} \mathrm{Re}$ in a total volume of 2-4 mL of lipiodol was instilled in the proper hepatic artery or in its right and/or left branches.

\section{Pathology of the Explanted Livers}

Total hepatectomy specimens were transected in 1-cm-thick slices and examined by a senior pathologist. Macroscopically, sampling from the explanted liver was focused on nodules corresponding to a tumor localization on magnetic resonance imaging (MRI) and, routinely, of largersized regenerative nodules in the other segments. Diagnosis of HCC was made on histology, depending on light-microscopical characteristics of cellular growth pattern and nuclear atypia. The number of HCC lesions and their size, differentiation, percent of necrosis, and presence of microvascular invasion was noted.

\section{Assessment of Long-Term Outcome}

Follow-up after transplantation was made by control of tumor markers and imaging every 3 months. Survival was calculated from the day of transplantation until the date of the last followup visit or until death. 


\section{RESULTS}

Between June 1999 and June 2004, 37 patients, with biopsy-proven HCC or lesions suspect for $\mathrm{HCC}$ on radiology, were awaiting liver transplantation and received neoadjuvant radiolabeled lipiodol treatment at the Ghent University Hospital (Ghent, Belgium) (Fig.1). Nine patients who received other anticancer treatment modalities prior to radionuclide therapy were excluded from further analysis. Twenty-eight transplant candidates were exclusively pretreated by radiolabeled lipiodol. The mean age was 62 (range, 24-72) years, and the group consisted of 24 males versus 4 females. The vast majority suffered underlying viral hepatitis, and, to a lesser extent, alcohol-induced cirrhosis. Three and 21 patients underwent 1 administration of, respectively, ${ }^{188} \mathrm{Re}-\mathrm{HDD} /$ lipiodol and ${ }^{131}$ I-lipiodol. Two patients underwent 2 sessions of ${ }^{131}$ I-lipiodol and 2 patients received $3{ }^{131}$ I-lipiodol administrations. In 18 patients, both lobes were treated independent from lesion localization and in 10 patients only the affected lobe was treated. The median interval between the last radiolabeled lipiodol administration and the transplantation was 101 days (range, 32-320).

\section{Dropout Ratio on the Waiting List for Transplantation}

Two patients underwent a cadaveric transplantation without being listed according to the classic eligibility criteria (so called "special request" cadaveric transplantations), and 6 patients received a split liver from an adult living donor and, therefore, the number of patients at risk to drop out for transplantation was 20 . The average waiting time for classic cadaveric transplantation was 5.8 months. Two of 20 patients on the waiting list did not undergo liver transplantation because of death caused by nononcologic reasons and, thus, the drop-out ratio was $10 \%$ overall or $1.7 \%$ per month in the subgroup exclusively pretreated by radiolabeled lipiodol.

\section{Pathological Examination of the Explanted Livers}

The diagnosis of HCC was confirmed by histological examination of the explant in 22 of 26 cases. In 4 explants, the diagnosis of HCC could not be confirmed, as no lesions suspect for neoplastic disease were found. In these 4 cases, the diagnosis of $\mathrm{HCC}$ was based on the presence of hypervascular lesions on imaging which did not

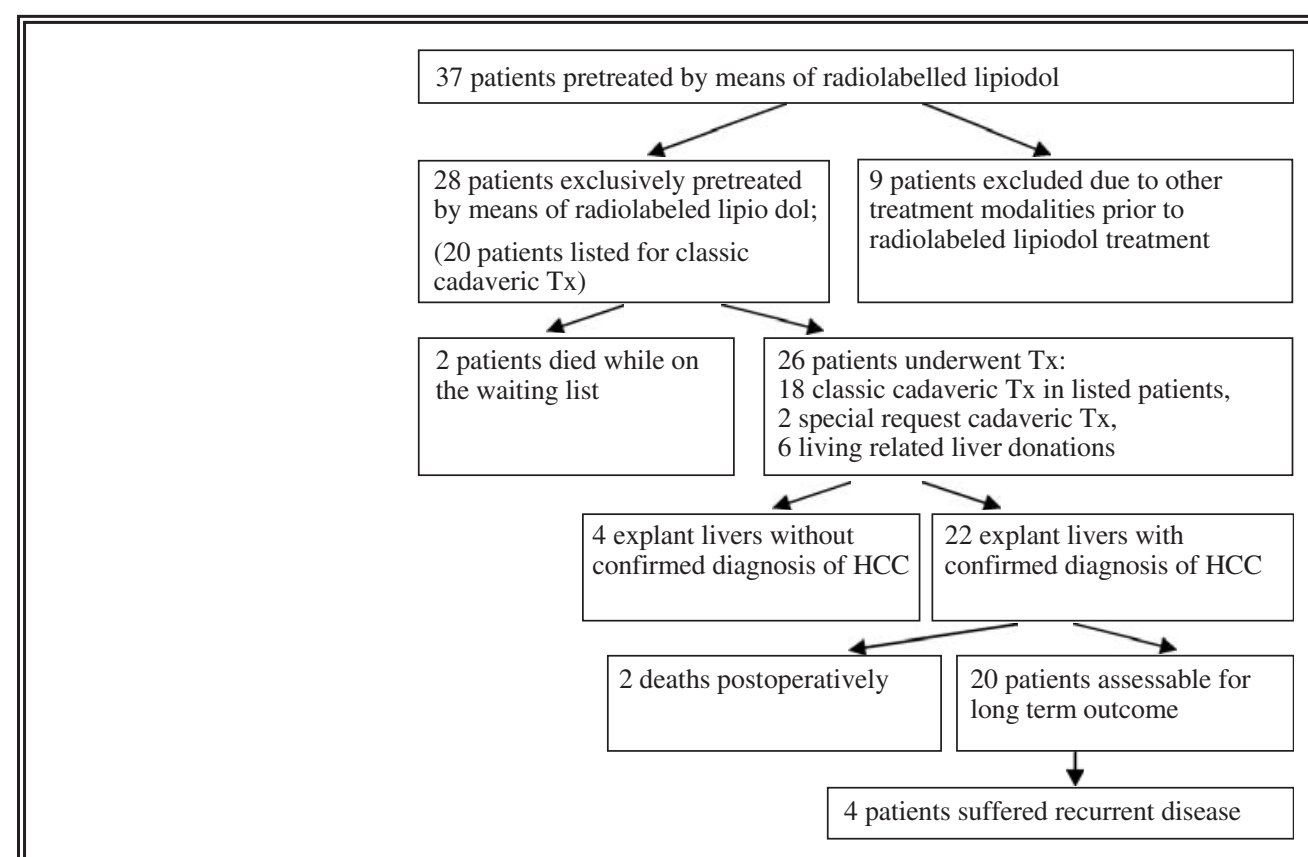

Figure 1. Flowchart depicting the evolution of 37 patients treated by means of radiolabeled lipiodol while awaiting liver transplantation (Tx). 
exceed the critical diameter of $2 \mathrm{~cm}$ and, hence, the diagnosis is debatable and these patients were excluded from further analysis. Light-microscopical findings in 22 assessable explants are summarized in Table 1.

\section{Long-Term Follow-Up}

Two patients died in the perioperative phase because of surgical complications. Four of 20 patients with a histological diagnosis of HCC (20\%), suffered recurrent disease after a mean disease-free interval of 6.9 months (range, 1.75-16). One patient suffered recurrent intrahepatic disease. Two patients developed pulmonary metastasis several months following transplantation, and one patient was diagnosed with cutaneous metastasis. The overall recurrence-free survival was 19.7 months (range, 1.75-56), with a mean follow-up of 20.1 months. The estimated risk for recurrence was $11.9 \%$ per patient year. Six patients received a liver transplantation, although their tumour load exceeded the criteria concerning size and number of the tumors described by Mazzaferro et al., and among these patients only 1 case of recurrent disease was observed. $^{4}$

\section{DISCUSSION}

Encouraging survival data on liver transplantation for treatment of early HCC have emerged in the last decade. However, the shortage of donor organs and the increasing demand cause waiting times of more than 6 months in some parts of Eu-

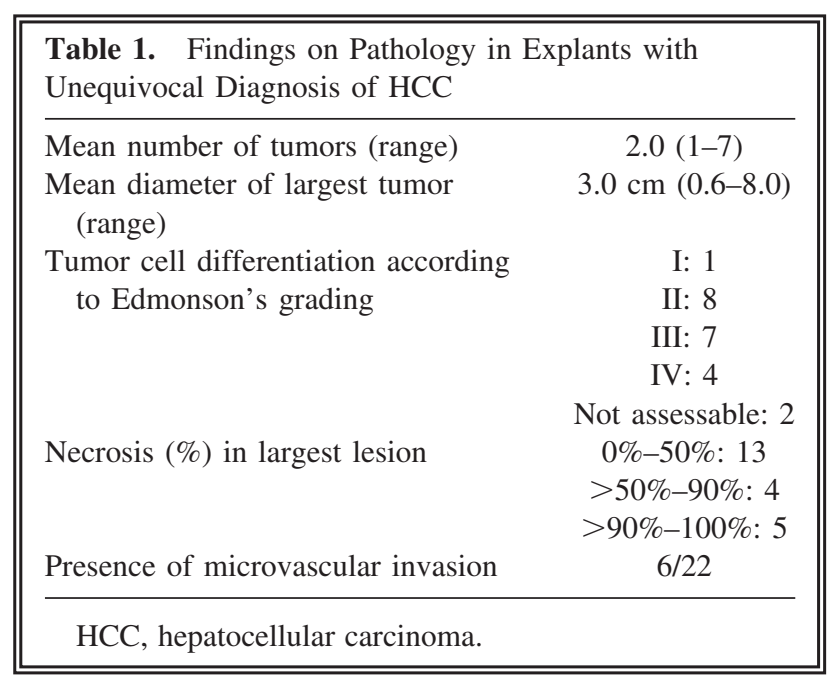

rope and the US. Several authors have shown that waiting times exceeding 6 months are associated with a more than $15 \%$ dropout rate from the waiting list, and dropout rates may reach $50 \%$ if expanded criteria are applied. ${ }^{14}$ In general, the risk for dropout while awaiting liver transplantation is assumed to be approximately $4 \%$ per month. ${ }^{15}$ Even after correction for the 4 cases without histological diagnosis of HCC, a considerably lower dropout ratio (2.2\% per month, or $12.5 \%$ over 5.8 months) was obtained in our series.

Four of 26 assessable explants (15\%) did not reveal any neoplastic, nor completely necrotic, lesion. In these patients, the preoperative findings did not fulfil the classic diagnostic criteria of $\mathrm{HCC}$; hence, the diagnosis of HCC remains questionable. Earlier explant assessment reports have shown that false-positive diagnosis, despite stateof-the-art imaging occurs in $9 \%-31 \%$ of transplant candidates, depending on the tumor stage. ${ }^{16}$ It is unclear whether a complete response following radionuclide therapy occurred in our series or whether the exposition to ionizing irradiation should have been avoided. In 22 explants with clear evidence of HCC, at least $90 \%$ of the tumor was necrotic in $23 \%$ of the explants. This compares favorably with the findings in a historical control group, consisting of 19 patients transplanted at our institution with HCC without pretreatment $(n=12)$ or with an incidental finding of a HCC in the explant $(n=7)$. Although these patients had comparable tumor characteristics, none showed $90 \%$ or more tumor necrosis.

The mean follow-up time of 20.1 months is acceptable, as most recurrences are expected to occur within the 1st year following surgery, and, to a lesser extend, within the 2 nd year. ${ }^{17}$

It is difficult to compare our series in terms of recurrence rates and survival with other reports, as most series vary in patient characteristics and some series comprise incidental HCC, which is thought to have a better prognosis, while others have focused on more advanced disease stages. ${ }^{6,12,14} \mathrm{Re}$ ports dating from the early 1990s often comprise patients with advanced disease stages, and recurrence rates following transplantation were as high as $54 \%$. More recent data suggest recurrence rates of $4 \%-16 \%$ following transplantation, applying restrictive selection criteria. ${ }^{18}$ In our series, lessstringent criteria for transplantation were used, and this is probably reflected in the recurrence rate of $20 \%$. An additional important drawback for assessing the impact of strategies for patients await- 
ing liver transplantation is the lack of controlled studies. ${ }^{18}$ Moreover, the long-term results should be based on an intention to treat analysis, and the true role of neoadjuvant treatment may be preventing dropout in those patients who wait for long periods until transplantation. ${ }^{8}$

To date, no firm evidence for improved longterm outcome is available for any adjuvant or neoadjuvant treatment strategy. ${ }^{18}$ Some evidence was established for the use of polyprenoic acid and ${ }^{131}$ I-lipiodol following partial liver resection in prospective randomized trials. ${ }^{19,20}$ In transplant patients, no firm evidence is provided yet, and future research should attempt to elucidate the role of local, locoregional, and systemic modalities. It is assumed that tumor cells are present in blood in microscopic quantities and, therefore, it seems reasonable to add systemic treatment during the immediate postoperative phase. Systemic treatments with limited toxicity, such as polyprenoic acid, are probably useful to test patients at risk to relapse within the 1st year following transplantation. Combined with aggressive local or locoregional treatment modalities while on the waiting list, this may yield an improved long-term outcome if based on an intention-to-treat analysis.

Following ${ }^{131}$ I-lipiodol treatment, patients were not eligible for surgery for 4 weeks because of radioprotection issues related to the relatively long physical half-life of ${ }^{131} \mathrm{I}$ ( 8 days). In patients with aggressive tumors, repeated radionuclide therapies were indicated and this led to unacceptable long intervals, during which the patients were not eligible for curative treatment. Because of the high energy of the gamma-ray emission of ${ }^{131} \mathrm{I}(365 \mathrm{keV})$ and its physical half-life of 8 days, a hospitalization up to 7 days is indicated according to the local radioprotective legislation. Considering the limited success of ${ }^{131}$ I-lipiodol for the treatment of relatively large tumors, the switch towards ${ }^{188} \mathrm{Re}$, a radionuclide with a higher energy of the beta-emission $(2120 \mathrm{keV}$ and $1960 \mathrm{keV}$ for ${ }^{188} \mathrm{Re}$ versus $606 \mathrm{keV}$ for ${ }^{131} \mathrm{I}$ ) might yield improved response rates. As of November 2002, we switched to ${ }^{188} \mathrm{Re}-\mathrm{HDD} /$ lipiodol and the use of ${ }^{188} \mathrm{Re}$, with its physical half-life of 17 hours, avoids most of the radioprotection problems and allows for a shorter hospitalization, as well as further dose escalations. ${ }^{21}$ Following ${ }^{188} \mathrm{Re}-\mathrm{HDD} / \mathrm{lipiodol}$ treatment, patients were eligible for transplantation after an interval of only 1 week.

\section{CONCLUSIONS}

The dropout ratio in this patient group exclusively pretreated by means of radiolabeled lipiodol, was $10 \%$ and after correction for cases without unequivocal diagnosis of HCC on the explant liver, a dropout ratio from the waiting list of $12.5 \%$ or $2.2 \%$ per month was calculated.

On pathology, nearly complete or complete necrosis was observed in $23 \%$ of assessable explants. Four of 20 patients $(20 \%)$ suffered from recurrent disease. The overall recurrence-free survival was 19.7 months (range, 1.75-56) with a mean follow-up of 20.1 months. Our data support the evaluation on larger patient numbers to confirm the benefit of radiolabeled lipiodol in candidates for liver transplantation suffering from HCC.

\section{ACKNOWLEDGMENTS}

This work was supported by a grant from the Bijzonder Onderzoeksfonds (Ghent University, Ghent, Belgium, No. 011D9501). We would like to thank J. M. Jeong from the Seoul National University (Seoul, Korea) for providing the HDDkits.

\section{REFERENCES}

1. Parkin MD, Bray F, Ferlay J, et al. Estimating the world cancer burden: Globocan 2000. Int J Cancer 2001; 94:153.

2. Schafer DF, Sorrell MF. Hepatocellular carcinoma. Lancet 1999;353:1253.

3. Little SA, Fong Y. Hepatocellular carinoma: Current surgical management. Semin Oncol 2001;28:474.

4. Mazzaferro V, Regalia E, Doci R, et al. Liver transplantation for the treatment of small hepatocellular carcinoma in patients with cirrhosis. $N$ Engl $\mathrm{J} \mathrm{Med}$ 1996;334:697.

5. Figueras J, Jaurrieta E, Valls C, et al. Hepatology 1997; $25: 1485$

6. Haug CE, Jenkins RL, Rohrer RJ, et al. Liver transplantation for primary hepatic cancer. Transplantation 1992;53:376.

7. Llovet JM, Burroughs A, Bruix J. Hepatocellular carcinoma. Lancet 2003;362:1907.

8. Wall WJ, Marotta PJ. Surgery and transplantation for hepatocellular cancer. Liver Transpl 2000;6:S16.

9. Llovet JM, Mas X, Aponte JJ, et al. Cost-effectiveness of adjuvant therapy for hepatocellular carcinoma during the waiting list for liver transplantation. Gut 2002;50:123. 
10. Raoul JL, Guyader D, Bretagne JF, et al. Prospective randomized trial of chemoembolization versus intra-arterial injection of 131-I-iodized oil in the treatment of hepatocellular carcinoma. Hepatology 1997;26:1156.

11. Brans B, De Winter F, Defreyne L, et al. The antitumoral activity of neoadjuvant intra-arterial ${ }^{131}$ I-lipiodol treatment for hepatocellular carcinoma: A pilot study. Cancer Biother Radiopharm 2001;16:333.

12. Raoul JL, Messner M, Boucher E, et al. Preoperative treatment of hepatocellular carcinoma with intra-arterial injection of I131-labeled lipiodol. Br J Surgery 2003;90:1379.

13. Lee YS, Jeong JM, Kim YJ, et al. Synthesis of ${ }^{188} \mathrm{Re}-$ labeled long chain alkyl diaminedithiol for therapy of liver cancer. Nucl Med Commun 2002;23:237.

14. Fisher RA, Maluf D, Cotterell AH, et al. Nonresective ablation therapy for hepatocellular carcinoma: Effectiveness measured by intention-to-treat and drop-out from liver transplant waiting list. Clin Transpl 2004; 18:502.

15. Sarasin FP, Majno PE, Llovet JM, et al. Living donor liver transplantation for early hepatocellular carcinoma:
A life-expectancy and cost-effectiveness perspective. Hepatology 2001;33:1073.

16. Wiesner RH, Freeman RB, Mulligan DC. Liver transplantation for hepatocellular carcinoma: The impact of the MELD allocation policy. Gastroenterology 2004; 127:S261.

17. Nonami T, Isshiki K, Katoh H, et al. The potential role of postoperative hepatic artery chemotherapy in patients with high-risk hepatomas. Ann Surg 1991;213:222.

18. Schwartz M. Liver transplantation for hepatocellular carcinoma. Gastroenterology 2004;127:S268.

19. Muto Y, Moriwaki H, Ninomiya M, et al. Prevention of second primary tumors by an acyclic retinoid, polyprenoic acid, in patients with hepatocellular carcinoma. N Engl J Med 1996;334:1561.

20. Lau WY, Leung TW, Ho SK, et al. Adjuvant intra-arterial iodine-131-labeled lipiodol for resectable hepatocellular carcinoma: A prospective randomised trial. Lancet 1999;353:797.

21. Lambert B, Bacher K, Defreyne L, et al. ${ }^{188}$ Re-HDD/ lipiodol therapy for hepatocellular carcinoma: A phase I clinical trial. J Nucl Med 2005;46:60. 